\title{
FUNCIONALIDADE E READMISSÃO HOSPITALAR EM PACIENTES COM AVC ISQUÊMICO
}

\author{
Larissa Borsa Lago'; Rodrigo Targa Martins²
}

\section{RESUMO}

O AVC é uma das principais causas de mortalidade e incapacidade adquirida no mundo. Ainda, dentre os sobreviventes, a readmissão hospitalar é comum. Este estudo objetivou analisar a relação entre funcionalidade e readmissões hospitalares em até um ano após a alta hospitalar em pacientes com AVC isquêmico submetidos à trombólise. Trata-se de um estudo de coorte retrospectivo. Foram coletados dados demográficos, epidemiológicos, NIHSS e Escala de Rankin modificada (mRS). Dos 48 pacientes incluídos na análise, a idade média foi de $60,4 \pm 12,91$. A freqüência de readmissão hospitalar totalizou 15 pacientes $(31,3 \%)$. Não foi observada diferença estatisticamente significativa quanto à idade $(p=0,982)$, sexo $(p=0,531)$, NIHSS $(p$ $=0,114)$ e $\mathrm{mRS}(\mathrm{p}=0,108)$ entre os pacientes readmitidos. Foi observada diferença entre o tempo de permanência na primeira internação e a readmissão hospitalar ( $p=$ $0,01)$. A causa mais freqüente de readmissão foram alterações comportamentais $(27,77 \%)$.

Palavras-chave: Classificação Internacional de Funcionalidade, Incapacidade e Saúde; Fisioterapia; Neurologia; Pesquisa.

Eixo Temático: Atenção Integral e Promoção à Saúde

\section{INTRODUÇÃO}

O AVC é uma das maiores causas de morte e incapacidade adquirida no mundo. Trata-se de uma doença dispendiosa devido ao grande número de mortes prematuras, incapacidade contínua em muitos sobreviventes, repercussão para as famílias ou prestadores de assistência, bem como para os serviços de saúde (WORLD HEALTH ORGANIZATION, 2006). Assim, é necessária uma melhor compreensão dos prejuízos funcionais após o evento isquêmico. Especificamente em pacientes com AVC, a Escala de Rankin modificada (mRS) é amplamente

\footnotetext{
${ }^{1}$ Hospital Universitário de Santa Maria. E-mail: lariblago@yahoo.com.br

${ }^{2}$ Grupo Hospitalar Conceição. E-mail: targa@ghc.com.br
} 
utilizada com este objetivo (FERNANDES et al., 2012).

Dentre os sobreviventes, a readmissão hospitalar é comum, com taxas que variam de 20 a 27\% no primeiro ano (SACCO; HAUSER; MOHR, 1991; THORNGREN; WESTLING; NORRVING, 1990). A readmissão hospitalar é utilizada como indicador de qualidade e eficiência dos cuidados hospitalares em várias condições clínicas (MEDICARE PAYMENT ADVISORY COMMISSION et al., 2013). Além disso, traz ônus ao sistema de saúde e desconforto ao paciente e familiares (BORGES et al., 2008). No entanto, poucos estudos (COLEMAN et al., 2004; SMITH et al., 1996) tem avaliado a funcionalidade enquanto variável para readmissão hospitalar.

A trombólise endovenosa com ativador tissular de plasminogênio recombinante (rt-PA) é atualmente a terapêutica mais estudada e com maior evidência de benefício em promover recanalização arterial no AVC isquêmico (TEIXEIRA; DE DEUS SILVA; FERREIRA, 2004). Os critérios de inclusão para rt-PA são os seguintes: AVC isquêmico em qualquer território encefálico; possibilidade de se iniciar a infusão do rt-PA dentro de 4,5 horas do início dos sintomas; tomografia computadorizada (TC) de crânio ou ressonância magnética sem evidência de hemorragia e idade superior a 18 anos. Os critérios de exclusão para trombólise endovenosa são: uso de anticoagulantes orais com tempo de protrombina com Razão Normalizada Internacional $(\mathrm{RNI})>1,7$; uso de heparina nas últimas 48 horas com Tempo de Tromboplasmina Parcial Ativada (TTPA) elevado; AVC isquêmico ou traumatismo cranioencefálico grave nos últimos 3 meses; história pregressa de hemorragia intracraniana ou de malformação vascular cerebral; TC de crânio com hipodensidade precoce $>1 / 3$ do território da artéria cerebral média; pressão arterial sistólica $\geq 185 \mathrm{mmHg}$ ou pressão arterial diastólica $\geq 110 \mathrm{mmHg}$ (em 3 ocasiões, com 10 minutos de intervalo) refratária ao tratamento anti-hipertensivo; melhoria rápida e completa dos sinais e sintomas no período anterior ao início da trombólise; déficits neurológicos leves (sem repercussão funcional significativa); cirurgia de grande porte ou procedimento invasivo nos últimos 14 dias; punção lombar nos últimos 7 dias; hemorragia geniturinária ou gastrointestinal nos últimos 21 dias ou história de varizes esofagianas; punção arterial em local não compressível na última semana; 
coagulopatia com Tempo de Protrombina (TP) prolongado (RNI > 1,7), TTPA elevado ou plaquetas < 100.000/mm3; glicemia < 50mg/dl com reversão dos sintomas após a correção; evidência de endocardite ou êmbolo séptico, gravidez; infarto do miocárdio recente (3 meses - contraindicação relativa); suspeita clínica de hemorragia subaracnóidea ou dissecção aguda de aorta (BRASIL, 2013).

Diante disso, este estudo objetivou analisar a relação entre funcionalidade e readmissões hospitalares em até um ano após a alta hospitalar em pacientes com AVC isquêmico submetidos à trombólise. Especificamente, os objetivos secundários foram mensurar a gravidade do AVC, verificar o tempo de permanência hospitalar, analisar variáveis demográficas e estabelecer se alguma dessas variáveis tem correlação com readmissões hospitalares.

\section{METODOLOGIA}

Trata-se de um estudo observacional, de coorte e retrospectivo. Os dados referem-se a pacientes cuja primeira internação por AVC isquêmico se deu no período de janeiro de 2009 a setembro de 2012, num hospital público da cidade de Porto Alegre. Através do sistema informatizado disponível no local de estudo, foram analisados se houveram readmissões hospitalares em um ano após a alta hospitalar. Este estudo foi registrado na Plataforma Brasil e aprovado pelo Comitê de Ética e Pesquisa do Grupo Hospitalar Conceição, Projeto n 13-199, CAAE: 23115313.4.0000.5530. Em função do tipo de estudo houve dispensa de TCLE.

Foram coletados dados demográficos e epidemiológicos. A gravidade do AVC foi mensurada pela Escala de AVC do NIH (National Institute of Health Stroke Scale - NIHSS) e o comprometimento funcional foi avaliado na alta hospitalar através da Escala de Rankin modificada (mRS - modified Rankin Scale). O NIHSS é a escala mais utilizada para avaliação da gravidade e para acompanhamento da evolução clínica do AVC, enfatizando os tópicos mais eminentes do exame neurológico (HOSPITAL ISRAELITA ALBERT EINSTEIN, 2013). O NIHSS pode ter sua pontuação variando de 0 (sem evidência de déficit neurológico) a 42 (paciente em coma e irresponsivo). A Escala de Rankin modificada tem boa validade e confiabilidade entre examinadores (SLOT et al., 2008; WILSON et al., 2005) e 
mensura o comprometimento funcional em pacientes com AVC. A Escala de Rankin pontua de 0 a 6 , sendo 0 - Sem sintomas; 1 - Nenhuma incapacidade significativa, a despeito dos sintomas, e capaz de conduzir todos os deveres e atividades habituais; 2 - Leve incapacidade, incapaz de realizar todas as atividades prévias, porém é independente para os cuidados pessoais; 3 -Incapacidade moderada, requer alguma ajuda mas é capaz de caminhar sem assistência (pode usar bengala ou andador); 4 - Incapacidade moderadamente severa, incapaz de caminhar sem assistência e incapaz de atender às próprias necessidades fisiológicas sem assistência; 5 Deficiência grave, confinado à cama, incontinente, requerendo cuidados e atenção constante de enfermagem e 6 - Óbito. Assim como em outros estudos (FERNANDES et al., 2012; SLOT et al., 2008), foi definido como estado independente um $m R S \leq 2$, e um estado de dependência funcional como $m R S \geq 3$.

Para que fosse possível detectar uma diferença absoluta no percentual de readmissões hospitalares de no mínimo 30\%, considerando um poder de $80 \%$ e nível de significância de 0,05, foram necessários no mínimo 48 pacientes. Consideraram-se como critérios de inclusão: internação com diagnóstico médico de AVC isquêmico no período compreendido entre 2009 e 2012, e ter recebido terapia trombolítica. Foram excluídos do estudo pacientes com diagnóstico de AVC hemorrágico, Ataque Isquêmico Transitório (AIT) e pacientes cujas readmissões hospitalares se tratassem de reinternações programadas, tais como cirurgias eletivas. Foram excluídos ainda os pacientes que pontuassem o valor de 6 na Escala de Rankin modificada, uma vez que tais indivíduos não poderiam reinternar.

\section{RESULTADOS}

Foram analisados 52 pacientes, dos quais 4 foram excluídos por óbito (mRS 6). Dos 48 pacientes incluídos na análise (Tabela 1), houve predominância do sexo masculino ( $\mathrm{n}=27$ ), a idade média foi de $60,4 \pm 12,91$ e a média do NIHSS foi $10 \pm$ 4,91. Quanto ao tempo de internação, obteve-se mediana de 11 (percentil 25 e 75: 8 - 18). Na tabela 1 estão demonstrados a caracterização da amostra e específico para os grupos mRS baixo e alto. Observa-se que o grupo $\mathrm{mRS}$ alto obteve mediana 
de 17 (percentil 25 e 75: 9,5 - 29,5), demonstrando um tempo de internação significativamente maior do que no grupo mRS baixo $(p=0,006)$.

A frequência de readmissão hospitalar totalizou 15 pacientes $(31,3 \%)$. Não foi observada diferença estatisticamente significativa quanto à idade ( $p=0,982)$, sexo $(p=0,531)$, NIHSS $(p=0,114)$ e mRS $(p=0,108)$ entre os pacientes readmitidos. Foi observada diferença estatisticamente significativa entre o tempo de permanência na primeira internação e a readmissão hospitalar $(p=0,01)$, sendo que pacientes readmitidos tiveram mediana de tempo de internação mais elevada.

A causa mais frequente de readmissão foram alterações comportamentais (27,77\%, 5 readmissões). Complicações pulmonares, cardiovasculares, renais e novo AVC demonstraram as mesmas taxas de readmissões hospitalares $(16,66 \%, 3$ readmissões) e houve uma readmissão por alterações dermatológicas (5,55\%).

Tabela 1. Comparação entre os grupos mRS alto e mRS baixo.

\begin{tabular}{lcccc}
\hline & Total & mRS baixo & mRS alto & p \\
Sexo masculino & $56,3 \%^{\mathrm{a}}$ & $67,7 \%^{\mathrm{a}}$ & $35,3 \%^{\mathrm{a}}$ & \\
Idade & $60,4 \pm 12,91^{\mathrm{b}}$ & $58,55 \pm 13,29^{\mathrm{b}}$ & $63,76 \pm 11,83^{\mathrm{b}}$ & $0,184^{\mathrm{d}}$ \\
Tempo de internação & $11(8-18)^{\mathrm{c}}$ & $9(7-15)^{\mathrm{c}}$ & $17(9,5-29,5)^{\mathrm{c}}$ & $0,006^{\mathrm{e}}$ \\
NIHSS & $10 \pm 4,91^{\mathrm{b}}$ & $9,9 \pm 4,67^{\mathrm{b}}$ & $13,94 \pm 4,33^{\mathrm{b}}$ & $0,0049^{\mathrm{d}}$ \\
Readmissão & $15(31,3 \%)^{\dagger}$ & $7(22,6 \%)^{\mathrm{f}}$ & $8(47,1 \%)^{\mathrm{f}}$ & $0,108^{\mathrm{g}}$ \\
\hline
\end{tabular}

\section{DISCUSSÃO}

$\mathrm{Na}$ amostra de pacientes com AVC isquêmico submetidos à trombólise analisada no presente estudo, houve predominância do sexo masculino, divergente do obtido em estudos prévios nos Estados Unidos (FONAROW et al., 2011; BRAVATA et al., 2007) e Dinamarca (ANDERSEN et al., 2000), porém em concordância com os achados de estudos brasileiros (FERNANDES et al., 2012; ROLIM; MARTINS, 2011). Cabe ressaltar que, a despeito das semelhanças encontradas nos estudos de readmissão hospitalar no AVC, não foram encontrados estudos cuja metodologia incluísse unicamente pacientes submetidos à trombólise, 
que configura uma população mais homogênea do que as analisadas em outros estudos, e resultados divergentes podem ser atribuídos a essas diferenças de delineamento.

Em um estudo (FERNANDES et al., 2012) que investigou os preditores para dependência funcional após o AVC isquêmico, entre eles a escolaridade e o nível socioeconômico, foi encontrada uma proporção maior de pacientes considerados independentes pelo mRS (independentes: 60\%), semelhante aos resultados encontrados em nosso estudo (independentes/mRS baixo: 64,6\%). Obteve-se idade média considerada idosa para países em desenvolvimento, semelhante aos dados encontrados em um estudo brasileiro (ROLIM; MARTINS, 2011) que avaliou a qualidade do cuidado hospitalar prestado no AVC isquêmico no Sistema Único de Saúde (SUS). Para esta finalidade, neste estudo (ROLIM; MARTINS, 2011) foram utilizados os dados de mortalidade hospitalar precoce (até $07^{\circ}$ dia de internação), considerando o perfil de gravidade dos casos e a realização de tomografia computadorizada. Um estudo brasileiro (FERNANDES et al., 2012) e um norteamericano (FONAROW et al., 2011) obtiveram populações com idade média ainda mais avançada, considerada idosa para países desenvolvidos.

Nossos resultados quanto à média do NIHSS foram mais elevados que os de um estudo (FONAROW et al., 2011) que incluiu 91.134 pacientes beneficiários do Medicare e quantificou as taxas de readmissão hospitalar, mortalidade em 30 dias e em 1 ano após o AVC isquêmico. Porém, em nosso estudo o NIHSS foi avaliado em todos os pacientes que compunham a amostra, enquanto no estudo norte-americano (FONAROW et al., 2011) o NIHSS foi documentado em apenas $37,4 \%$ dos pacientes, além de tratar-se de uma amostra diferenciada, cuja população constava de pacientes com AVC isquêmico, mas não necessariamente submetidos à terapia trombolítica como em nosso estudo. Ainda, este dado pode advir de uma maior gravidade do AVC em nossa amostra de pacientes.

Obteve-se menor taxa de readmissão hospitalar quando comparada a dois estudos norte-americanos (FONAROW et al., 2011; BRAVATA et al., 2007). Um deles (BRAVATA et al., 2007), que buscou determinar as taxas de readmissão hospitalar e de mortalidade, bem como as causas para a readmissão, por um 
período de 5 anos após o AVC, em indivíduos beneficiários do Medicare com idade $\geq$ 65 anos, obteve taxa de readmissão de 53,3\%. Já no estudo citado no parágrafo anterior (FONAROW et al., 2011), a taxa de readmissão foi de 56,2\%. Porém, nossos resultados quanto à readmisssão hospitalar foram semelhantes ao encontrado em outros três estudos, sendo dois conduzidos nos Estados Unidos, com taxas de 32,5\% (BOHANNON; LEE, 2003) e 37,3\% (BOHANNON; LEE, 2004), e um em Cingapura, que obteve 37,4\% (SUN; TOH, 2009) de readmissão. Cabe ressaltar que os dois estudos (FONAROW et al., 2011, BRAVATA et al., 2007) que obtiveram taxas de readmissão mais elevadas restringiram-se à população beneficiária pelo programa governamental estadunidense Medicare, que assiste a uma população mais específica, especialmente idosos e pessoas com incapacidades, o que já propiciaria índices mais elevados para readmissões hospitalares. Os três estudos (BOHANNON; LEE, 2003; BOHANNON; LEE, 2004; SUN; TOH, 2009) que obtiveram taxas semelhantes a nossa avaliaram populações mais heterogêneas.

Nos resultados do presente estudo, não houve diferença estatisticamente significativa na comparação entre sexo e readmissão hospitalar, assim como em outros estudos (BOHANNON; LEE, 2003; BOHANNON; LEE, 2004; HELLER et al., 2000), tendo sido encontrado apenas um único trabalho (KENNEDY, 2005) em que foi reportada esta variável com associação significativa para readmissão hospitalar. Porém, o estudo realizado na Califórnia (KENNEDY, 2005) sugeriu que haveria um risco de readmissão mais elevado no sexo feminino apenas na faixa etária de 15 a 54 anos de idade, uma população distinta da analisada em nosso trabalho, na qual predominaram indivíduos idosos.

Em um ensaio clínico randomizado (ANDERSEN et al., 2000) que avaliou dois modelos de acompanhamento pós-alta hospitalar em pacientes com incapacidade persistente após AVC foi identificada idade elevada como variável independente para risco de readmissão, no entanto tratou-se de uma amostra com maiores níveis de dependência funcional do que a avaliada em nosso estudo. Assim como o encontrado em estudos norte-americanos (BOHANNON; LEE, 2003; BOHANNON; LEE, 2004), para nossa amostra não houve diferença estatisticamente significativa 
na correlação entre idade e readmissão hospitalar. Estes estudos avaliaram mortalidade, readmissão hospitalar e a associação destes desfechos com as variáveis tempo de permanência na primeira internação, idade e NIHSS (BOHANNON; LEE, 2003); e a freqüência de readmissão e o valor preditivo da funcionalidade para este desfecho (BOHANNON; LEE, 2004).

Assim como em outros estudos (BOHANNON; LEE, 2003; BOHANNON; LEE, 2004), não foi encontrada associação entre NIHSS e readmissão hospitalar. Possivelmente, tal dado tenha relação com a baixa sensibilidade do NIHSS para AVCs da circulação posterior e à tendência da escala a subestimar a extensão da lesão no hemisfério cerebral direito em relação ao esquerdo (KRIEGER et al., 1999).

Não houve associação entre maior nível de dependência funcional e readmissão hospitalar, divergente dos resultados encontrados na literatura (BOHANNON; LEE, 2004; CHUANG et al., 2005). Entretanto, tais pesquisas (BOHANNON; LEE, 2004; CHUANG et al., 2005) mensuraram a funcionalidade com métodos diferentes do utilizado em nosso estudo, uma vez que nossa avaliação se deu pela Escala de Rankin modificada, e o estudo norte-americano (BOHANNON; LEE, 2004) utilizou o Índice de Barthel, enquanto o trabalho realizado em Taiwan (CHUANG et al., 2005) analisou o número de limitações em Atividades de Vida Diária (AVD). Além disso, estes estudos contaram com uma amostra maior, especificamente 228 (BOHANNON; LEE, 2004) e 489 (CHUANG et al., 2005) pacientes. Assim, o menor poder de nossa pesquisa, dado o número de pacientes avaliados, provavelmente foi responsável pela ausência de diferença estatisticamente significativa nesta comparação.

Encontrou-se uma associação significativa entre maior tempo de permanência hospitalar na primeira internação e readmissão hospitalar. Um estudo de coorte realizado em Taiwan (TSENG; LIN, 2009), que avaliou readmissão em um ano após AVC em 468 pacientes também obteve associação significativa para esta variável. Ainda, a literatura traz que o tempo de internação está diretamente relacionado ao número de complicações, à gravidade do comprometimento neurológico e à organização do atendimento (ARBOIX et al., 1994; HEUSCHMANN et al., 2004 ). 
A causa de readmissão hospitalar mais freqüente foram alterações comportamentais, porém os dados na literatura são conflitantes quanto às causas de readmissão no AVC isquêmico: em dois estudos norte-americanos que avaliaram mortalidade e readmissão hospitalar de beneficiários do programa Medicare, um deles (BRAVATA et al., 2007) obteve como categoria diagnóstica mais comum associada à readmissão pneumonia ou doenças respiratórias, com uma taxa de readmissão anual entre $8,2 \%$ e 9,0\%, enquanto no outro estudo (FONAROW et al., 2011), do total de readmissões, $73,7 \%$ foram atribuídas a causas não cardiovasculares. Cabe ressaltar novamente que tratam-se de populações distintas da analisada no presente estudo, uma vez que situaram-se em um país com desenvolvimento socioeconômico mais elevado que o Brasil e foram estudados apenas os usuários do programa do governo federal norte-americano Medicare. Além disso, a divisão para causas de readmissão hospitalar foi menos específica que a metodologia que adotamos.

\section{CONCLUSÃO}

As limitações deste estudo se devem à amostra reduzida, ao delineamento retrospectivo, mais sujeito a vieses, e a não correção das variáveis quanto às comorbidades. Outras pesquisas investigando readmissão hospitalar em pacientes com AVC isquêmico devem ser conduzidas, com maiores amostras, e analisando também outros fatores, como redes de apoio social, fatores socioeconômicos e diferentes escalas de funcionalidade.

\section{REFERÊNCIAS}

ANDERSEN, H. E. et al. Can readmission after stroke be prevented? Results of a randomized clinical study: a postdischarge follow-up service for stroke survivors. Stroke, v. 31, n. 5, p. 1038-1045, 2000.

ARBOIX, A. et al. Mortalidad en la fase aguda de la enfermedad cerebrovascular: registro de La Alianza - Hospital Central de Barcelona. Medicina clinica, v. 103, n. 14, p. 529-533, 1994. 


\section{QUFN}

BOHANNON, R. W.; LEE, N. Association of physical functioning with samehospital readmission after stroke. American journal of physical medicine \& rehabilitation, v. 83, n. 6, p. 434-438, 2004.

BOHANNON, R. W.; LEE, N. Hospital readmissions and deaths during the first year after hospitalization for stroke. Connecticut medicine, v. 67, n. 9, p. 535-539, 2003.

BORGES, F. K. et al. Reinternação hospitalar precoce: avaliação de um indicador de qualidade assistencial. Revista HCPA. Porto Alegre. Vol. 28, n. 3 (2008), p. 147-152, 2008.

BRASIL. Ministério da Saúde. Secretaria de Atenção à Saúde. Departamento de Atenção Especializada. Manual de rotinas para atenção ao AVC. Brasília: Editora do Ministério da Saúde, 2013.

BRAVATA, D. M. et al. Readmission and death after hospitalization for acute ischemic stroke: 5-year follow-up in the medicare population. Stroke, v. 38, n. 6, p. 1899-1904, 2007.

CHUANG, K.-Y. et al. Identifying factors associated with hospital readmissions among stroke patients in Taipei. Journal of nursing research, v. 13, n. 2, p. 117128, 2005.

COLEMAN, E. A. et al. Posthospital care transitions: patterns, complications, and risk identification. Health services research, v. 39, n. 5, p. 1449-1466, 2004.

FERNANDES, T. G. et al. Educational levels and the functional dependence of ischemic stroke survivors. Cadernos de saude publica, v. 28, p. 1581-1590, 2012. 
FONAROW, G. C. et al. Hospital-level variation in mortality and rehospitalization for medicare beneficiaries with acute ischemic stroke. Stroke, v. 42, n. 1, p. 159166, 2011.

HELLER, R. F. et al. Death and readmission in the year after hospital admission with cardiovascular disease: the Hunter Area Heart and Stroke Register. Medical journal of Australia, v. 172, n. 6, p. 261-265, 2000.

HEUSCHMANN, P. U. et al. Factors influencing duration of hospitalization after stroke in Germany. Deutsche medizinische Wochenschrift (1946), v. 129, n. 7, p. 299-304, 2004.

HOSPITAL ISRAELITA ALBERT EINSTEIN. Diretrizes Assistenciais - Acidente Vascular Cerebral. Disponível em: http://medsv1.einstein.br/diretrizes/neurologia /AVC.pdf. Acessed November, 22, 2013.

KENNEDY, B. S. Does race predict stroke readmission? An analysis using the truncated negative binomial model. Journal of the National Medical Association, v. 97, n. 5, p. 699, 2005.

KRIEGER, D. W. et al. Early clinical and radiological predictors of fatal brain swelling in ischemic stroke. Stroke, v. 30, n. 2, p. 287-292, 1999.

MEDICARE PAYMENT ADVISORY COMMISSION et al. Report to the Congress: promoting greater efficiency in Medicare. Medicare Payment Advisory Commission (MedPAC), 2007.

ROLIM, C. L. R. C.; MARTINS, M. Qualidade do cuidado ao acidente vascular cerebral isquêmico no SUS. Cadernos de Saúde Pública, v. 27, p. 2106-2116, 2011. 
SACCO, R. L.; HAUSER, W. A.; MOHR, J. P. Hospitalized stroke in blacks and Hispanics in northern Manhattan. Stroke, v. 22, n. 12, p. 1491-1496, 1991.

SLOT, K. B. et al. Impact of functional status at six months on long term survival in patients with ischaemic stroke: prospective cohort studies. Bmj, v. 336, n. 7640, p. 376-379, 2008.

SMITH, D. M. et al. Risk factors for nonelective hospital readmissions. Journal of general internal medicine, v. 11, n. 12, p. 762-764, 1996.

SUN, Y.; TOH, M. P. H. S. Impact of diabetes mellitus (DM) on the health-care utilization and clinical outcomes of patients with stroke in Singapore. Value in Health, v. 12, p. S101-S105, 2009.

TEIXEIRA, R. A.; DE DEUS SILVA, L.; FERREIRA, V. Tratamento trombolítico no acidente vascular cerebral isquêmico. Revista Neurociências, v. 12, n. 1, p. 5-17, 2004.

THORNGREN, M.; WESTLING, B.; NORRVING, B. Outcome after stroke in patients discharged to independent living. Stroke, v. 21, n. 2, p. 236-240, 1990.

TSENG, M.-C.; LIN, H.-J. Readmission after hospitalization for stroke in Taiwan: results from a national sample. Journal of the neurological sciences, v. 284, n. 1-2, p. 52-55, 2009.

WILSON, J. T. et al. Reliability of the modified Rankin Scale across multiple raters: benefits of a structured interview. Stroke, v. 36, n. 4, p. 777-781, 2005.

WORLD HEALTH ORGANIZATION. WHO STEPS stroke manual: the WHO STEPwise approach to stroke surveillance. Geneva: World Health Organization; 2006. 